IMMUNODEFICIENCY

\section{Natural killers need NEMO}

The natural killer (NK) cells of patients who have a defect in the $\mathrm{NF}-\kappa \mathrm{B}$ pathway are not up to the job, according to a new study in The Journal of Clinical Investigation. But, the good news is that treatment with interleukin-2 (IL-2) can restore their killer potential.

Hypohidrotic ectodermal dysplasia with immunodeficiency (HED-ID) is an X-linked syndrome that results from mutations in the NEMOgene - which encodes inhibitor of $\kappa \mathrm{B}$ kinase- $\gamma(\mathrm{IKK}-\gamma)$. IKK- $\gamma$ is essential for the formation of a functional IKK complex, which phosphorylates $I \kappa B \alpha$; this allows the translocation of NF- $\kappa B$ to the nucleus and gene transcription. The immunodeficiency that is seen in patients with HED-ID is variable, but defects in both T- and B-cell responses have been reported.

Orange and colleagues studied three patients that had HED-ID; two of these were shown to have new mutations in NEMO. One of these patients had recurrent infections with cytomegalovirus (CMV), which is indicative of defective NK-cell activity. This led the authors to examine all three patients for NK-cell defects. The numbers of NK cells in the peripheral blood of these patients were within the normal range. However, the cytotoxic activity of the NK cells against tumour-cell targets in vitro- a classic measure of NK-cell function - was completely abolished. A control experiment showed that the patients' NK cells were effective in an antibody-dependent cellular cytotoxicity assay, which indicates that the cytotoxic effector machinery was intact.

Next, the authors investigated whether it might be possible to correct the NEMO-dependent NKcell defect. In vitro treatment with IL-2, which is a potent enhancer of NK-cell activity, restored the cytotoxic activity of NK cells from patients with HED-ID. An electrophoretic mobility-shift assay then showed that stimulation with IL-2 induced NF- $\mathrm{KB}$ activity in these NK cells. These in vitro findings provided the rationale for treating the patient who suffered from recurrent CMV infection with IL-2. Promisingly, ex vivo NK-cell cytotoxicity was apparent immediately and persisted for four weeks after treatment. So, in addition to defining a new molecular pathway in NK-cell activity, this study highlights a potential therapy to restore antiviral innate immunity in HED-ID patients.

Jennifer Bell

(2) References and links ORIGINAL RESEARCH PAPER Orange, J. S. et al. Deficient natural killer cell cytotoxicity in patients with IKK- $\gamma / \mathrm{NEMO}$ mutations. J. Clin. Invest. 109 1501-1509 (2002)

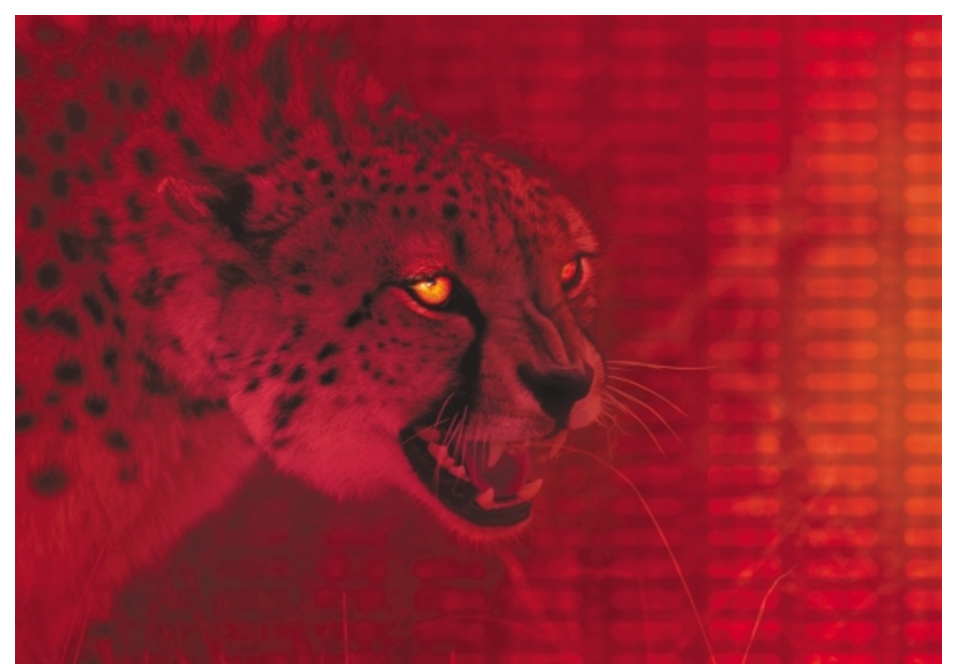

\section{IN BRIEF}

\section{T-CELL SIGNALLING}

Induction of T helper type 2 immunity by a point mutation in the LAT adaptor.

Aguado, E. et al. Science 296, 2036-2040 (2002)

\section{A LAT mutation that inhibits T-cell development yet} induces lymphoproliferation.

Sommers, C. L. et al. Science 296, 2040-2043 (2002)

The adaptor protein LAT (linker for activation of T cells) links the T-cell receptor (TCR) to downstream signalling effectors. Two groups have now generated mice that are homozygous for a mutation of a single LAT tyrosine residue (Tyr136) to investigate the role of this residue in vivo. Both groups report an early block in T-cell differentiation and the accumulation of polyclonal CD4 helper T cells in the periphery. Aguado et al. show that these $T$ cells produce large quantities of type- 2 cytokines, which results in tissue eosinophilia and the secretion of IgE and IgG1 by plasma cells. Sommers et al. analysed TCR signalling and showed that the activation of PLC- $\gamma$ and NFAT, calcium signalling, IL-2 production and cell death are defective in these mice. These studies highlight the crucial role of LAT in T-cell development and homeostasis.

\section{TRANSPLANTATION}

Allogeneic $\beta$-islet cells correct diabetes and resist immune rejection.

Pericin, M. et al. Proc. Natl Acad. Sci. 99, 8203-8206 (2002)

Usually, allogeneic MHC-incompatible cell grafts are rejected rapidly by immunocompetent hosts. Here, Pericin et al. successfully correct streptozotocin-induced diabetes by transplanting fully MHC-mismatched insulin-producing $\beta$-islet cells under the kidney capsule of recipient mice. This was made possible by the generation of a growth-regulated, transformed endocrine cell line that could be transplanted into recipients without contaminating passenger leukocytes. The grafts that controlled hyperglycaemia were not rejected for $>100$ days.

\section{T-CELL RESPONSES}

Programmed contraction of CD8 ${ }^{+} \mathrm{T}$ cells after infection. Badovinac, V. P., Porter, B. B. \& Harty, J. T. Nature Immunol. 3, 619-626 (2002)

Normally, the contraction phase of a T-cell response is correlated with clearance of the pathogen, but are these events mechanistically linked? This study indicates that for $\mathrm{CD} 8^{+} \mathrm{T}$ cells, the contraction phase is actually pre-programmed and, unlike the expansion phase, it is not dependent on the continued presence of available antigen. The kinetics of the contraction phases of $\mathrm{CD}^{+} \mathrm{T}$-cell responses to viral or bacterial infection in mice were shown to be independent of the quantity of available antigen, duration of infection or magnitude of T-cell clonal expansion. These results indicate that the contraction of CD8 ${ }^{+}$ $\mathrm{T}$-cell populations is programmed to occur even if a pathogen has not been cleared successfully. 